\title{
The regulation of ferroptosis by MESH1 through the activation of the integrative stress response
}

\author{
Chao-Chieh $\operatorname{Lin}^{1,2,5}$, Chien-Kuang Cornelia Ding ${ }^{1,2,5}$, Tianai Sun ${ }^{1,2}$, Jianli Wu ${ }^{1,2}$, Kai-Yuan Chen ${ }^{3}$, Pei Zhou ${ }^{4}$ and Jen-Tsan Chi $\mathbb{D}^{1,2}{ }^{凶}$
}

(c) The Author(s) 2021

All organisms exposed to metabolic and environmental stresses have developed various stress adaptive strategies to maintain homeostasis. The main bacterial stress survival mechanism is the stringent response triggered by the accumulation "alarmone" ( $p$ ) ppGpp, whose level is regulated by RelA and SpoT. While metazoan genomes encode MESH1 (Metazoan SpoT Homolog 1) with ppGpp hydrolase activity, neither ppGpp nor the stringent response is found in metazoa. The deletion of Mesh1 in Drosophila triggers a transcriptional response reminiscent of the bacterial stringent response. However, the function of $M E S H 1$ remains unknown until our recent discovery of MESH1 as the first cytosolic NADPH phosphatase that regulates ferroptosis. To further understand whether MESH1 knockdown triggers a similar transcriptional response in mammalian cells, here, we employed RNA-Seq to analyze the transcriptome response to MESH1 knockdown in human cancer cells. We find that MESH1 knockdown induced different genes involving endoplasmic reticulum (ER) stress, especially ATF3, one of the ATF4-regulated genes in the integrative stress responses (ISR). Furthermore, MESH1 knockdown increased ATF4 protein, elF2a phosphorylation, and induction of ATF3, XBPs, and CHOP mRNA. ATF4 induction contributes to $30 \%$ of the transcriptome induced by MESH1 knockdown. Concurrent ATF4 knockdown re-sensitizes MESH1-depleted RCC4 cells to ferroptosis, suggesting its role in the ferroptosis protection mediated by MESH1 knockdown. ATF3 induction is abolished by the concurrent knockdown of NADK, implicating a role of NADPH accumulation in the integrative stress response. Collectively, these results suggest that MESH1 depletion triggers ER stress and ISR as a part of its overall transcriptome changes to enable stress survival of cancer cells. Therefore, the phenotypic similarity of stress tolerance caused by MESH1 removal and NADPH accumulation is in part achieved by ISR to regulate ferroptosis.

Cell Death and Disease (2021)12:727; https://doi.org/10.1038/s41419-021-04018-7

\section{INTRODUCTION}

Nutrient deprivation and various forms of environmental stresses impact the physiology and survival of organisms. To maintain homeostasis and survival under metabolic stresses, all organisms have developed adaptive strategies to cope with these adverse conditions. When bacteria are confronted with low nutrient availability, these metabolic stresses lead to the accumulation of guanosine $3^{\prime}, 5^{\prime}$-bidiphosphate (pppGpp or ppGpp), which triggers the 'stringent response' as a distinct metabolic state $[1,2]$. (p) ppGpp binds to RNA polymerase and dramatically alters transcriptome to mediate a stress survival expression program. In addition, (p)ppGpp also inhibits translation initiation by binding to the IF2 (initiation factor 2) [3]. The levels of (p)ppGpp are regulated by its synthesis (by RelA) and degradation (by SpoT) $[1,2]$. While the stringent response is highly conserved among bacteria and plants, no stringent response has been reported in mammals [4].

In multi-cellular organisms, the microenvironments of most cells are usually maintained within narrow physiological ranges. However, such "normal" physiological conditions can be disrupted by various disease processes, including vascular blockage during cardiac ischemia or stroke that would lead to a rapid depletion of oxygen, glucose as well as accumulation of lactic acids and other metabolic wastes. Similarly, the microenvironments of solid tumors may also exhibit glucose and amino acid deprivation, hypoxia, and lactic acidosis, which are caused by defective blood vessels, poor vascular perfusion, and overgrowth of the tumor cells. In mammalian cells, different stress conditions lead to distinct stress responses to coping with specific metabolic challenges. For example, under limiting oxygen, cells develop a hypoxia response triggered by hypoxia-inducible factors that mediate various metabolic changes to cope with the limited oxygen supply [5-7]. Similarly, lactic acidosis from tumor hypoxia and anaerobic glycolysis trigger metabolic reprogramming to limit glycolysis [8-11]. Glucose deprivation leads to energy depletion, increased AMP/ATP ratios, and subsequent AMPK activation that limits biosynthesis through the phosphorylation of acetyl-CoA carboxylase 1 (ACC1) [12]. Over time, these stress conditions also select tumors with genetic changes that offer survival advantages under stresses $[10,13]$. Other than these stress-specific responses, mammalian cells also have a conserved and stereotypic stress response pathway named the endoplasmic reticulum (ER) stress or

\footnotetext{
${ }^{1}$ Department of Molecular Genetics and Microbiology, Duke University Medical Center, Durham, NC, USA. ${ }^{2}$ Duke Center for Genomic and Computational Biology, Durham, NC,

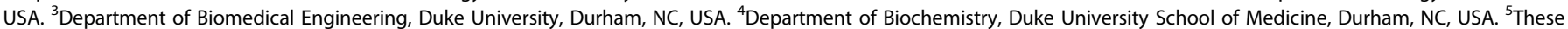
authors contributed equally: Chao-Chieh Lin, Chien-Kuang Cornelia Ding. ${ }^{\circledR}$ email: jentsan.chi@duke.edu Edited by B. Zhivotovsky
} 
unfolding protein response (UPR). ER stress can be initiated by amino acid deprivation [14], hypoxia, and lactic acidosis [10] as well as other stresses [15]. ER stress or UPR are usually divided into three parallel and distinct branches as defined by signaling proteins in ER: PERK (PKR-like ER kinase), IRE1 (inositol requiring enzyme 1), and activating transcription factor 6 (ATF6). A PERK branch mediates an integrative stress response (ISR) [15], which is characterized by the phosphorylation of the elF2a (alpha subunit of eukaryotic translation initiation factor 2) that reduces the global protein synthesis while allowing the preferential translation of ATF4. ATF4 functions to induce the transcription of another transcription factor, CHOP (GADD153/DDIT3). Together, ATF4 and CHOP enhance the transcriptional expression of genes involved in amino acid metabolism and resistance to oxidative stress.

Although the stringent response has not been reported in metazoa or mammals, the metazoan genome contains MESH1 (Metazoan homologs of SpoT), a homolog of bacterial SpoT that possesses the enzymatic activities of the ppGpp hydrolase [16]. However, (p)ppGpp is barely detectable in metazoan cells. The genetic deletion of Mesh1 in Drosophila shows upregulation of stress-responsible genes, suggesting the presence of a bacterial stringent-like stress response in metazoa [16]. Recently, we identified MESH1 as the first cytosolic NADPH phosphatase [17] which was induced by erastin treatment or cystine deprivation [18]. MESH1 knockdown robustly protected cells from ferroptosis, a novel form of iron-dependent cell death characterized by lipid peroxidation $[18,19]$. Ferroptosis is triggered by a wide variety of environmental stresses experienced by mammalian cells. MESH1 was identified by the functional genomic screens during cystinedeprived death [20]. Therefore, similar to the SpoT inhibition to protect bacterial cells from stress death, MESH1 removal also protected human cells from ferroptosis induced by extreme oxidative stresses. While MESH1 removal leads to stress tolerance phenotypes, much remains unknown about the transcriptional response of $M E S H 1$ removal.

The transcriptional response is a prominent feature of a bacterial stringent response. Since the deletion of Mesh1 in Drosophila led to transcriptional response reminiscent of bacterial stringent response [16] and the knockdown of MESH1 conferred robust protection against ferroptosis [18], we wished to understand the transcriptional response to MESH1 knockdown in human cells. Here we report that as part of the transcriptional response to MESH1 knockdown, it triggers a prominent feature of the ER stress response with repression of cell proliferation and the ATF4 activation by ISR contributing to ferroptosis protection under MESH1 knockdown. These findings suggest MESH1 removal triggers a stress response that helps mammalian cells to cope with extreme oxidative stresses and prevents ferroptosis.

\section{RESULTS \\ MESH1-knockdown induces an extensive transcriptional response}

The bacterial stringent response is characterized by extensive transcriptome changes [1]. Similarly, the depletion of Drosophila Mesh1 also triggered dramatic transcriptional changes reminiscent of bacterial stringent response [16]. Since the knockdown of human $\mathrm{MESH} 1$ offered strong ferroptosis protection, we wished to investigate the transcriptional response to MESH1 knockdown to (1) understand the ferroptosis protection phenotypes and (2) explore their potential similarity with these previously reported features of bacterial stringent response by removal of Drosophila Mesh1. We employed RNA-Seq to profile the transcriptome of RCC4 cells transfected with either control or two independent MESH1 siRNAs that efficiently knocked down MESH1 RNA expression (GEO: GSE114282). The successful knockdown of MESH1 was confirmed in the RNA-Seq data (Fig. 1A). The transcriptome analysis revealed that $\mathrm{MESH} 1$ knockdown triggered extensive transcriptional responses, including the downregulation of several genes in the DNA synthesis and cell cycle progression, including CDK2 (Cyclin-dependent kinase 2), E2F1 (E2F transcription factor 1), and RRM2 (Ribonucleotide Reductase Regulatory Subunit M2) (Fig. 1A). Next, the Gene Ontology (GO) analysis confirmed that $M E S H 1$ knockdown repressed pathways associated with cell cycle progression and DNA replication (Fig. 1B, Supplementary Tables 1 and 2). Gene set enrichment analysis (GSEA) revealed that MESH1 knockdown repressed DNA replication, which was also one of the features of stringent response in Escherichia coli [21] (Fig. 1C, Supplementary Tables 1 and 2). In addition, MESH1 knockdown also repressed several gene sets associated with ribosome structural constituents and organelles, implying reduced translation (Fig. 1D). To validate the results of RNA-seq, we knocked down MESH1 by control or two independent MESH1 siRNA in RCC4 cells (Fig. 1E) and used quantitative real-time polymerase chain reaction ( $q R T-P C R$ ) to confirm the significant repression of cell cycle progression genes, including CDK2 (Fig. 1F), E2F1 (Fig. 1G), and RRM2 (Fig. 1H). RRM2 encodes a subunit of ribonucleotide reductase which provides the dNTPs required for DNA synthesis. Interestingly, the bacterial stringent response in E. coli and Mesh1-deficient Drosophila also caused similar cell cycle arrest [16, 21-23]. These data suggest that MESH1 knockdown in mammalian cells triggers a transcriptional response of cell cycle arrest and reduced ribosomal activities with a striking similarity to those observed in the bacterial stringent response [22, 23] and Mesh1 deficient Drosophila [16]. Among the transcriptional response (Fig. 1A), we are particularly interested in the upregulation of ATF3 (Fig. 1A), considering its role as a canonical target gene of the ISR, a PERK branch of the ER stress [15].

\section{MESH1 knockdown triggers an ER stress response}

The main function of the bacterial stringent response is to maintain survival and homeostasis under metabolic stresses and nutrient deprivations. One similar stress survival process in mammalian cells is ER stress [15] triggered by a wide variety of stress conditions. A PERK branch of ER stress, or ISR, is characterized by the elF2a phosphorylation, ATF4 protein stabilization, transcriptional induction of ATF3, and other ISR genes. We noted that $M E S H 1$ knockdown triggered the induction of ATF3 mRNAs by RNA-seq (Fig. 1A), a prominent feature of ISR. Therefore, we studied the changes of other ISR markers and noted the upregulation of other ISR genes, including the C/EBP homologous protein $(C H O P)$, and cystathionine gamma-lyase (CTH) (Fig. 1A). As elF2a phosphorylation is a feature of ISR, we extracted proteins from RCC4 cells transfected with control or two independent MESH1 siRNA and determined the status of elF2a phosphorylation using Western blots (Fig. 2A). MESH1 siRNAs successfully reduced the MESH1 protein levels (Fig. 2A) and increased the elF2a phosphorylation (Fig. 2A). Giving that elF2a phosphorylation reduces the global protein synthesis, this is consistent with the repressed ribosomal pathways at the transcriptome levels in the GSEA analysis (Fig. 1D). elF2a phosphorylation is often associated with increased ATF4 translation through the switching between different upstream ORFs [24]. Consistently, MESH1 knockdown increased the level of ATF4 protein (Fig. 2A), a key transcriptional regulator of the ISR known to activate ATF3, CTH, and CHOP. Therefore, after confirming the successful knockdown of MESH1 RNA (Fig. 2B), we found MESH1 knockdown induced the expression of $A T F 3$ and $C T H$ transcripts in RCC4 cells (Fig. 2C, D). Furthermore, we found that MESH1 knockdown also induced the upregulation of CHOP in RCC4 cells (Fig. 2E) and 786-O cells (Supplementary Fig. 1A, B). Together, these data suggest that MESH1 knockdown activates the PERK branch of ER stress pathway.

Besides PERK pathway, we further examined the potential of MESH1 knockdown to trigger the ATF6 and IRE1 branches of ER 
A
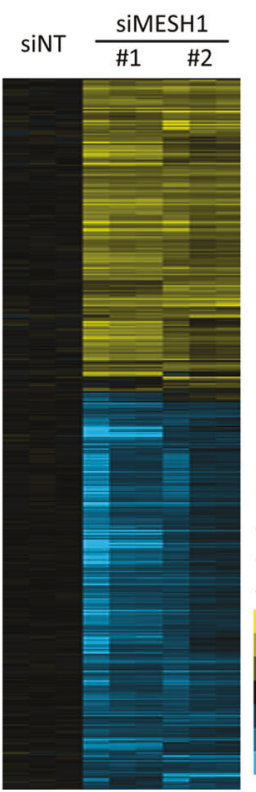

E

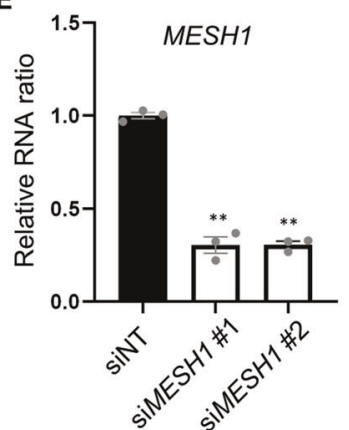

B

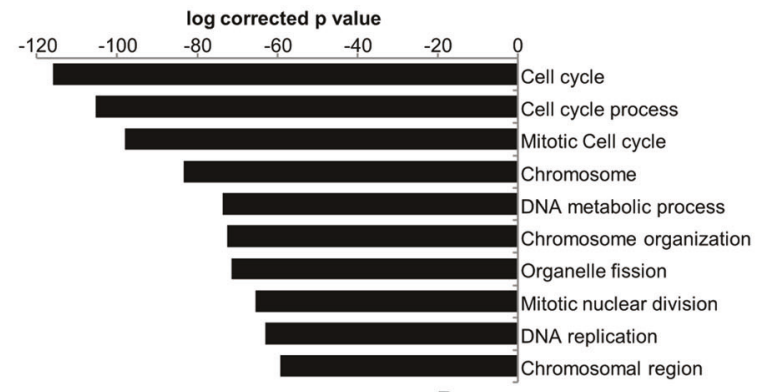

D

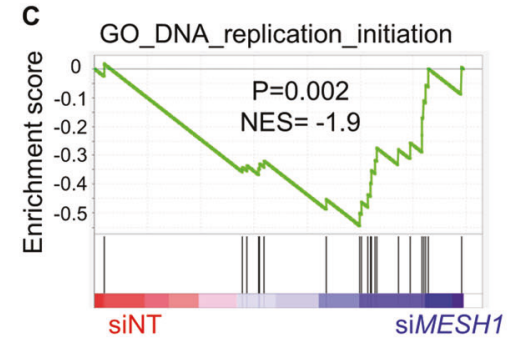

GO_Structural_constituent_of_ribosome

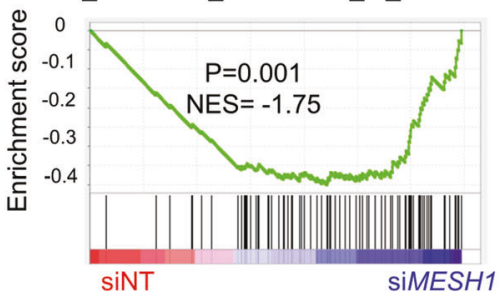

$\mathbf{F}$

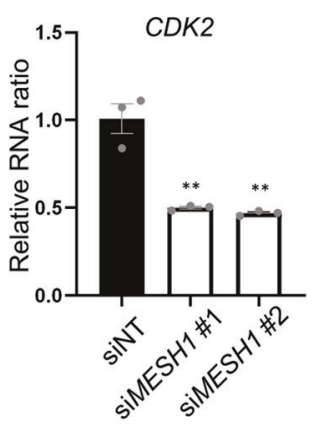

H

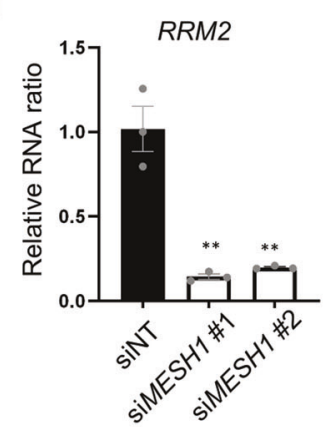

Fig. 1 MESH1 knockdown suppresses a transcriptional response of cell cycle progression. A Heatmap of the transcriptional difference between control or two independent MESH1 siRNA knockdown in RCC4 cells. MESH1 was silenced by siMESH1\#1 or siMESH1\#2 and profiled by RNA-seq. Color scale indicates log2-fold-change. B Top ten repressed Gene Ontology processes of the transcriptome of $M E S H 1$-silenced RCC4 cells. C, D GSEA analysis showed the enrichment of DNA replication geneset (C) and constituent of ribosome (D) in the MESH1 knockdown cells. E-H mRNA abundance of MESH1 (E), CDK2 (F), E2F1 (G), and RRM2 (H) as validated by rt-qPCR. mRNA abundance was determined by rt-qPCR, normalized by $\beta$-actin, and presented in relative ratio to siNT (non-targeting) treatment. $n=3$ biological replicates. Statistical analysis: ANOVA with Tukey HSD post hoc test, ${ }^{* *} P<0.01$.

stresses. MESH1 knockdown induced the expression of HERPUD1 (Fig. 2F) and XBP1 total mRNA expression (Fig. 2G), suggesting the activation of ATF6. The activated IRE1 catalyzes the excision of an intron from $X B P 1$-unspliced isoform $(X B P 1 u)$ to become $X B P 1$ spliced isoform (XBP1s). Therefore, the $X B P 1 s / X B P 1 u$ ratio reflects the IRE1 activity and determines the folding capacity in ER [25]. qRT-PCR revealed that $M E S H 1$ knockdown significantly increased the XBP1s/XBP1u ratio in $\mathrm{RCC} 4$ and $786-\mathrm{O}$ cells (Fig. $2 \mathrm{H}$, Supplementary Fig. 1C) as well as XBP1s target gene ERdj4 (Fig. 2I). Taken together, these data indicate that MESH1 knockdown actives all three branches of ER stress pathway.

\section{The role of ATF4 in the transcriptional response to MESH1 knockdown}

ATF4 is the main transcription factor responsible for the transcriptional feature of ISR [15]. To determine the contribution of ATF4 to the transcriptome response to MESH1 knockdown, we compared the transcriptional response of RCC4 to MESH1 knockdown alone or in combination with ATF4 knockdown (Fig. 3A, GEO: GSE114128). The heatmap shows that the MESH1 and ATF4 double knockdown mitigated a portion of the transcriptional response of MESH1 knockdown (Fig. 3A).
However, a significant portion of the transcriptional response of MESH1 knockdown was not affected by the ATF4 knockdown (Fig. 3A). In general, approximately 30\% (294 out of 980 genes) of the MESH1-knockdown signature was affected by simultaneous ATF4 knockdown (Fig. 3B), suggesting that ATF4-mediated integrated stress response is a notable feature of MESH1knockdown signature. Next, we used qRT-PCR to validate the ATF4-mediated vs. non-ATF4-mediated transcriptional response to MESH1 knockdown. We examined the RNA expression of two known target genes of ATF4, ATF3, and CTH (Fig. 3C, D). MESH1 knockdown upregulated $A T F 3$ and $C T H$, and these upregulations were indeed abolished by simultaneous ATF4 knockdown (Fig. 3C, D). We also validated the MESH1-knockdown responsive gene that was not affected by simultaneous ATF4 knockdown (Fig. 3E, F). We found that ACLY (ATP citrate synthase) (Fig. 3E) and RRM2 (Fig. 3F) were both repressed upon MESH1 knockdown, but these changes were not affected by simultaneous ATF4 knockdown (Fig. 3E, F). Collectively, these data suggest the ATF4-mediated transcriptional profile contributed significantly to a portion of the transcriptional response to MESH1 knockdown. However, MESH1 knockdown also triggered ATF4independent transcriptional changes. 


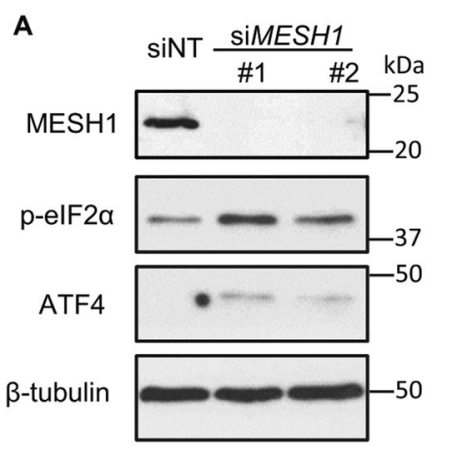

B

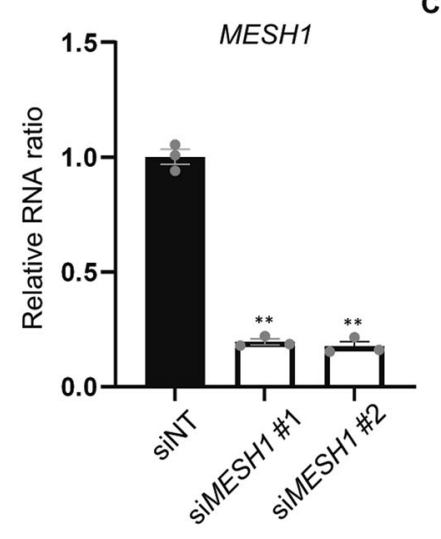

D

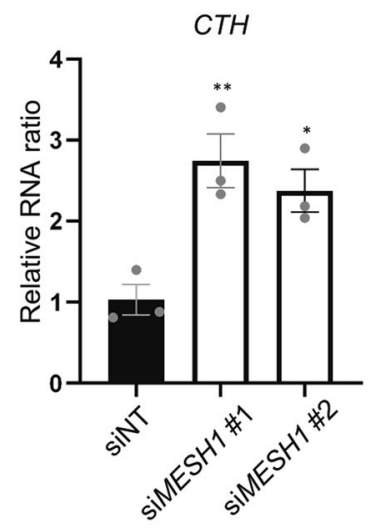

G

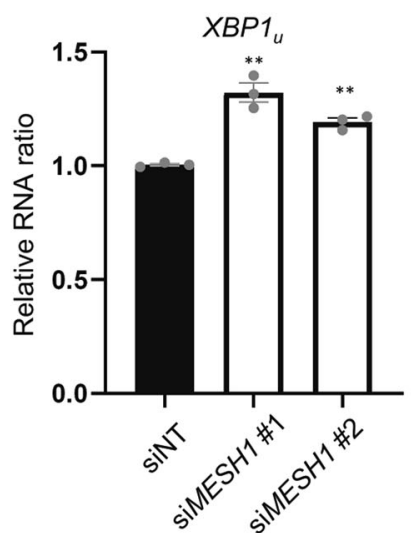

E

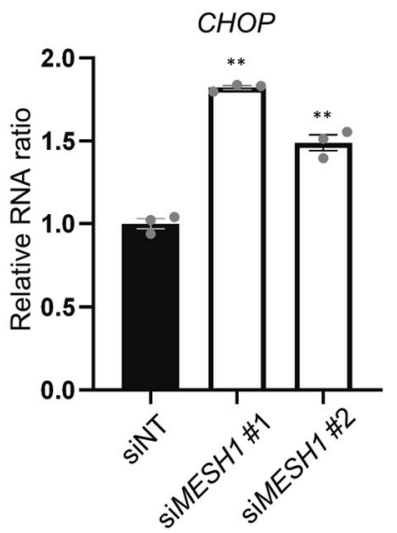

H

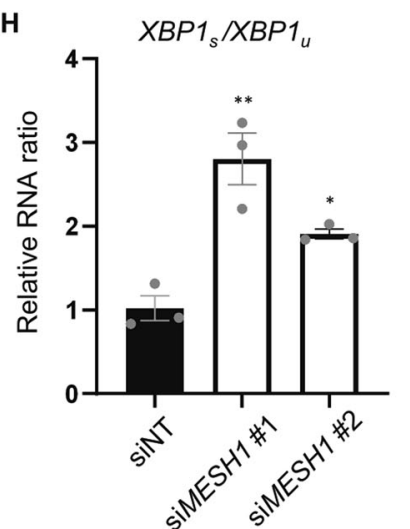

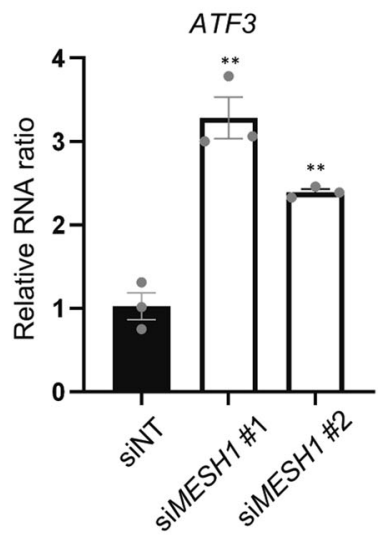

$\mathbf{F}$

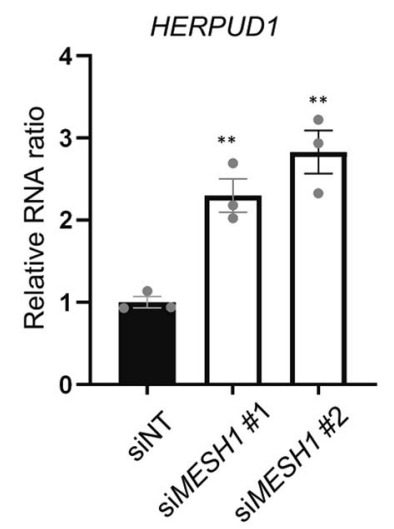

I

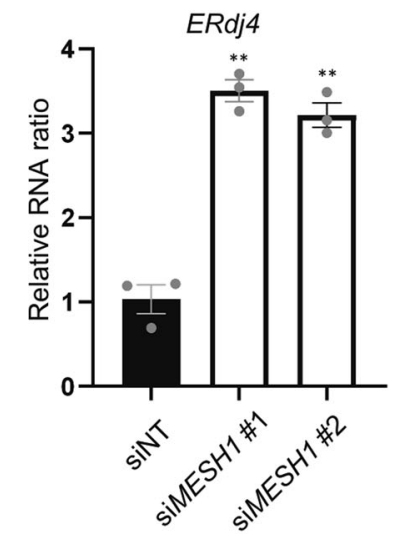

Fig. 2 MESH1 knockdown activates the ER stress response. A MESH1 knockdown increased phosphorylated elF2 $\alpha$ protein and ATF4 protein expression. RCC4 cells knocked down by non-targeting (NT) siRNA or two independent MESH 1 siRNA for three days were lysed for WB. $\beta$-actin as a loading control. B-I MESH1 knockdown (B) increased mRNA expression of several genes involving in the PERK pathway, including ATF3 (C), $C T H(\mathbf{D}), C H O P(\mathbf{E})$, ATF6 target genes: HERPUD1 (F), and total XBP1 (XBP1u) (G), IRE1 target gene: spliced XBP1(XBP1s) as determined by XBP1s/ $X B P 10$ ratio $(\mathbf{H})$ and ERdj4 (I). $n=3$ biological replicates. Statistical analysis: ANOVA with Tukey HSD post hoc test, ${ }^{*} P<0.05, * * P<0.01$.

The functional role of ATF4 and ISR in the MESH1 knockdown Previously, we found that MESH1 knockdown protects RCC4 cells from ferroptosis [18]. To investigate the role of ISR in the stringent responses induced by MESH1 knockdown, we first evaluated whether the activation of ISR can prevent ferroptosis. Given tunicamycin can inhibit the $\mathrm{N}$-linked glycosylation of proteins in ER to trigger ISR [26], we treated cells with erastin alone or together with tunicamycin (Fig. 4A). We found that the treatment of tunicamycin significantly protected cells from ferroptosis, consistent with previous reports [27-29] (Fig. 4A). Brefeldin A can also trigger ISR by targeting protein transport from ER to Golgi [30]. Similar to tunicamycin, brefeldin A also protected cells from erastin-induced ferroptosis (Fig. 4B). These data suggest that ISR triggered by tunicamycin or brefeldin can protect cells from ferroptosis. Given our data suggests that $\mathrm{MESH} 1$ depletion triggers ISR by upregulating ATF4 expression (Figs. 2 and 3), we determined whether the induced ATF4 may contribute to the ferroptosis protection offered by MESH1 knockdown. To test this possibility, we determined if simultaneous ATF4 knockdown would mitigate the ferroptosis protection phenotypes of MESH1 knockdown. Indeed, the ATF4 knockdown abolished the ferroptosis survival phenotype of MESH1-knockdown cells by the CellTiter Glo assay (Fig. 4C). We further validated the results using the CellTox Green assay to observe the membrane rupture under erastin 
A

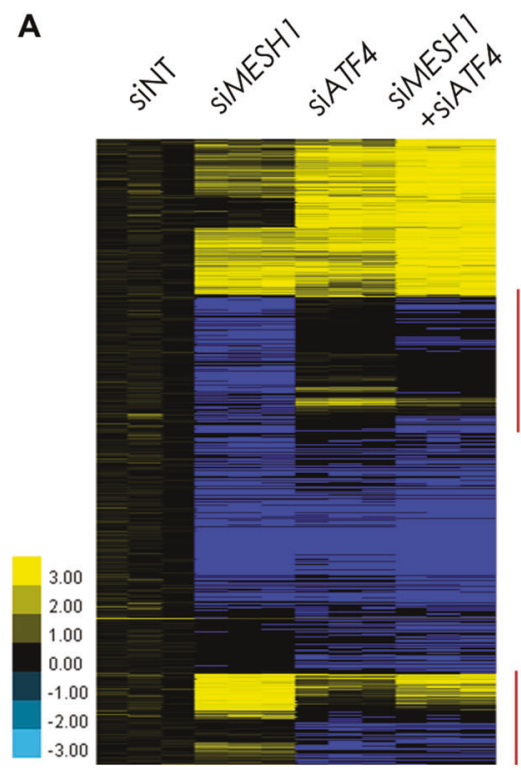

mediated

C

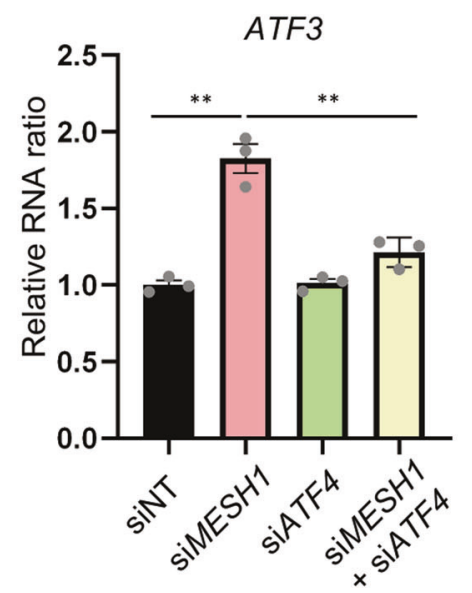

E

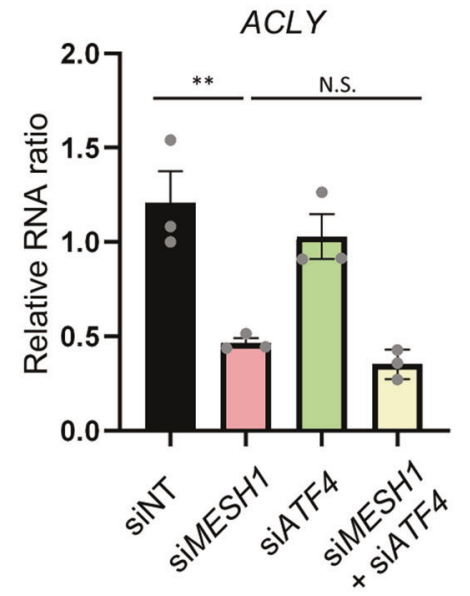

treatment (Fig. 4D) as quantified in Fig. 4E. In this assay, the dye enters the cells after membrane rupture and then binds DNA to display green fluorescent signals. We found that the protective effect of MESH1 knockdown under erastin treatment was
ATF4

\section{B}

\section{siMESH1 signature}

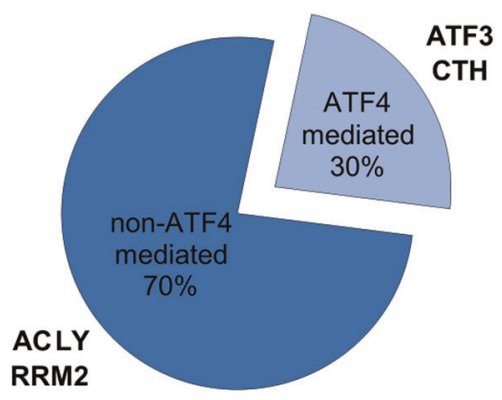

non-ATF4

mediated

D

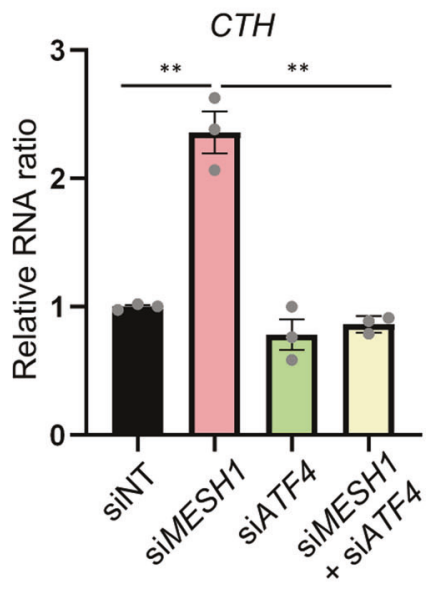

$\mathbf{F}$

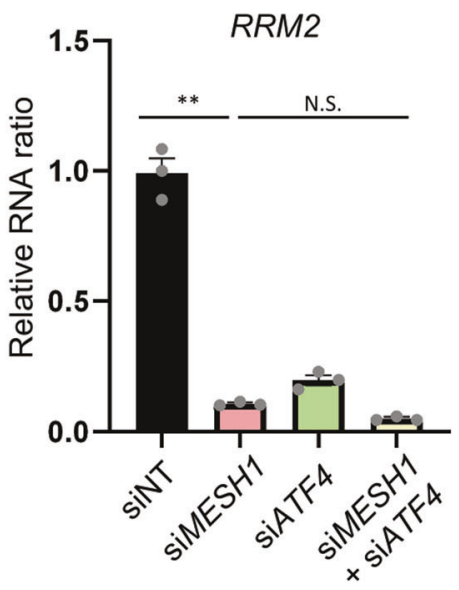

abolished by simultaneous ATF4 knockdown. These results indicate that the ferroptosis-resistance phenotype in MESH1knockdown cells may require the activation of the ATF4 and integrated stress response pathway. 
Fig. 3 Simultaneous ATF4 knockdown abolished a portion of MESH1-knockdown signature. A Heatmap of the transcriptional response of RCC4 to MESH1 knockdown alone or in combination with ATF4 knockdown profiled by the array. B Transcriptional signature of MESH1 silencing (siMESH1 signature) is defined as differential gene expression with fold change $>1.41$ with $t$ test $P<0.05$. ATF4-mediated genes are defined as having a siMESH1 signature that is reversed upon simultaneous ATF4-silencing with fold change $>1.41$ and $t$ test $P<0.05$ compared to $M E S H 1$ knockdown toward the direction of siNT samples. Note that genes affected by siATF4 alone are identified as an off-target effect (due to the low expression level of ATF4 in unstressed RCC4 cells) and are excluded from the analysis. C, D Simultaneous ATF4-knockdown abolished the ATF4targeted genes ATF3 (C) and CTH (D) induced by MESH1-knockdown. E, F Simultaneous ATF4-knockdown did not alter ACLY (E) and RRM2 (F) induced by MESH1 knockdown in RCC4 cells. mRNA abundance was determined by rt-qPCR, normalized by $\beta$-actin, and presented in relative ratio to non-targeting control (C-F). Statistical analysis: ANOVA with Tukey HSD post-hoc test, ${ }^{* *} P<0.01$.

A

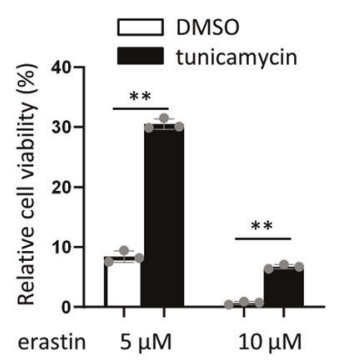

D

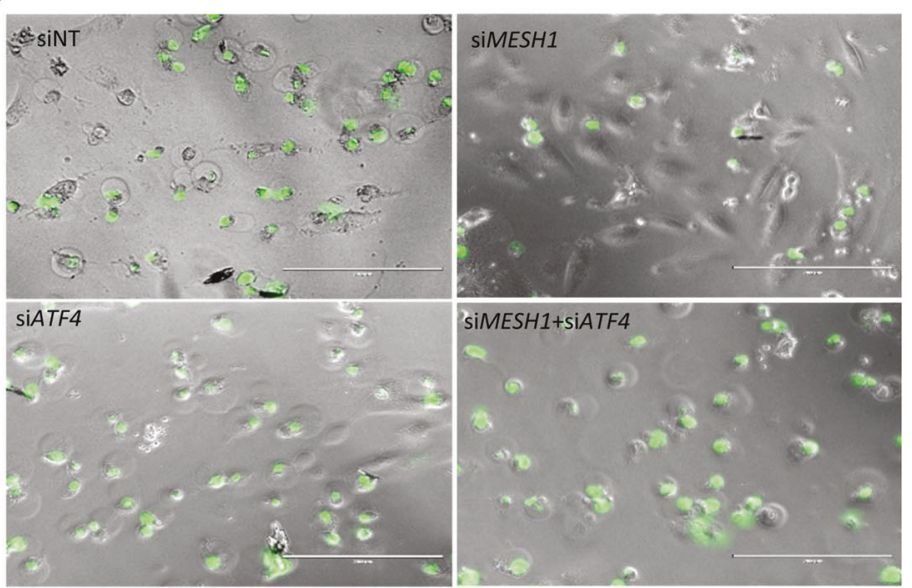

B

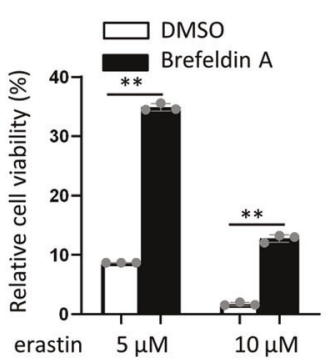

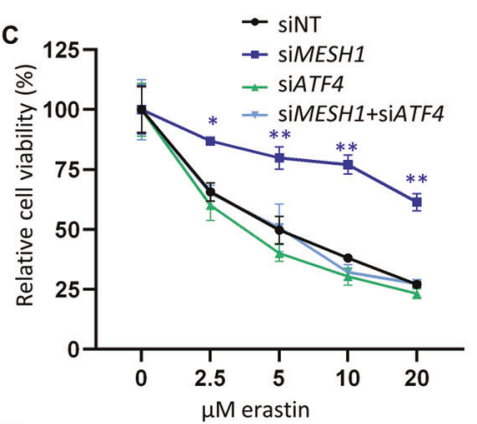

E

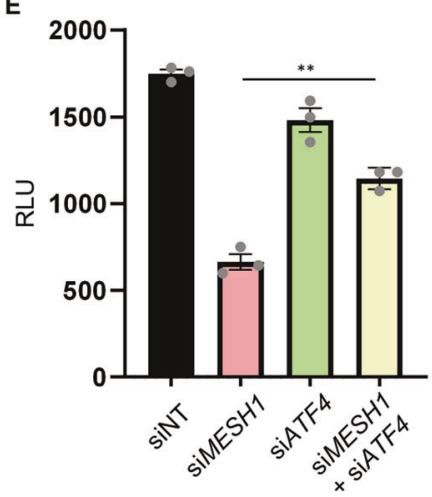

Fig. 4 ISR induced by MESH1 knockdown attenuates ferroptosis. A, B Tunicamycin and brefeldin A treatment prevents erastin-induced cell death. RCC4 cells were treated with erastin and in combination with tunicamycin $(6 \mu \mathrm{g} / \mathrm{ml})(\mathbf{A})$ or brefeldin $(2 \mu \mathrm{g} / \mathrm{ml})(B)$. The relative cell viability was determined by CellTiter Glo assay. C, D ATF4-knockdown abolished the ferroptosis survival phenotype of MESH1-knockdown in RCC4 cells as determined by CellTiter Glo assay (C) or CellTox Green assay to observe the membrane rupture under erastin treatment (5 $\mu M$ ) (D) and quantified in (E). Statistical analysis: ANOVA with Tukey HSD post hoc test, ${ }^{*} P<0.05,{ }^{*} P<0.01$.

\section{The potential contribution of enzymatic activities of MESH1 to the cellular responses to MESH1 knockdown}

We have previously found that MESH1 is an NADPH phosphatase that regulates the level of NADPH during the process of ferroptosis [18]. MESH1 mediates the dephosphorylation and degradation of cytosolic NADPH [18]. Importantly, the cytosolic NADPH can be generated by NAD kinase (NADK) by phosphorylating NADH [31]. Therefore, MESH1-depleted phenotypes, such as ferroptosis protection [32], were genetically "suppressed" by the knockdown of NADK through the suppression of the cytosolic NADPH level [18]. We thus transfected RCC4 cells with siRNA targeting MESH1 and $N A D K$, either alone or in combination for three days (Fig. 5A, $B)$, and examined the transcriptional changes. First, we validated two genes shown in our RNA-seq (Fig. 1A) to be regulated by the NADPH phosphatase activity of MESH1. Since the MESH1 was induced by extracellular nutrient (cystine) deprivation [18], we focused on genes that encoded proteins that modulate tumor angiogenesis and microenvironments, including vascular endothelial growth factor A (VEGFA) and Serpin Family E Member 1 (SERPINE1). We found that the downregulated VEGFA upon
MESH1 knockdown was mitigated by concurrent NADK knockdown (Fig. 5C). Also, the induction of SERPINE1 upon MESH1 knockdown was also mitigated by concurrent NADK knockdown (Fig. 5D). These data suggest that NADPH levels upregulated by MESH1 knockdown may regulate a subset of the transcriptional response. Therefore, we further examined whether the PERK pathway of ER stress was regulated similarly by measuring p-eIF2a and ATF4 protein levels by Western blots (Fig. 5E) and the mRNA levels of ATF3 (Fig. 5F), CHOP (Fig. 5G), and CTH (Fig. 5H). We found that the induction of different molecular features of the PERK pathway by MESH1 knockdown can be mitigated by the codepletion of NADK (Fig. 5E-H). Similar effects were also observed in ATF6 (XBP1 $1_{\mathrm{u}}$ ) (Fig. 5I) and IRE1a (XBP1 $1_{\mathrm{s}}$ ) (Fig. 5J) pathways. Collectively, these data implicate the role of the NADPH phosphatase activity of MESH1 in regulating ER stress. This is consistent with the previous observation that the knockdown of NADK mitigates the ferroptosis protection phenotypes associated with MESH1 knockdown [18]. Together, while our previous findings showed the crucial role of MESH1 as an NADPH phosphatase in regulating the NADPH level and ferroptosis, these 
A

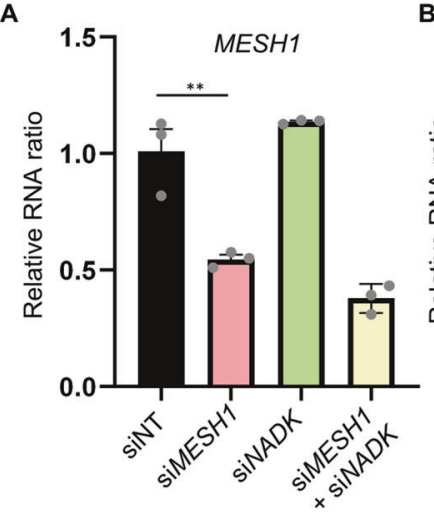

E

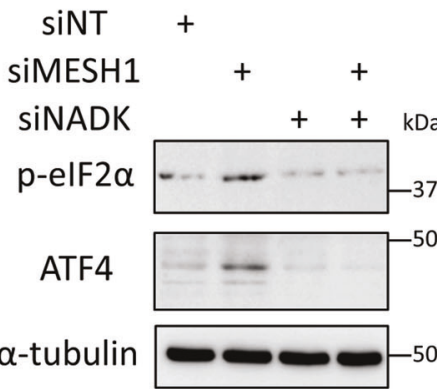

I

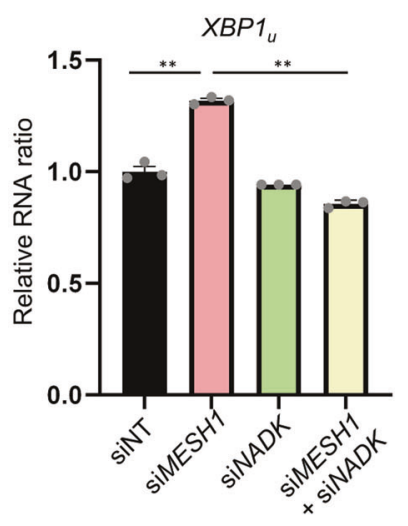

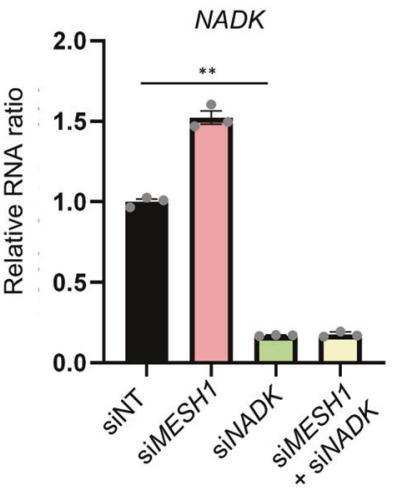

$\mathbf{F}$
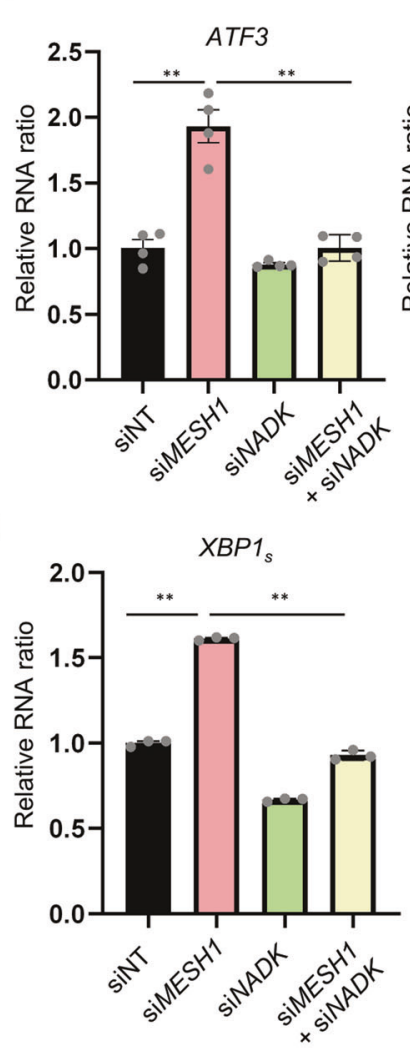

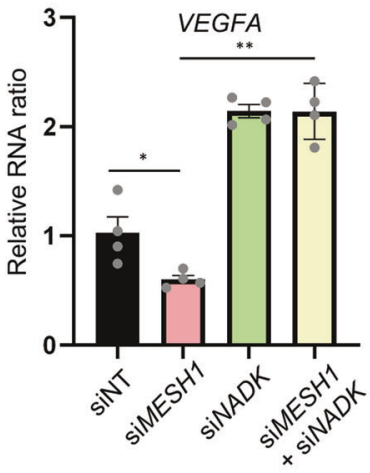

G

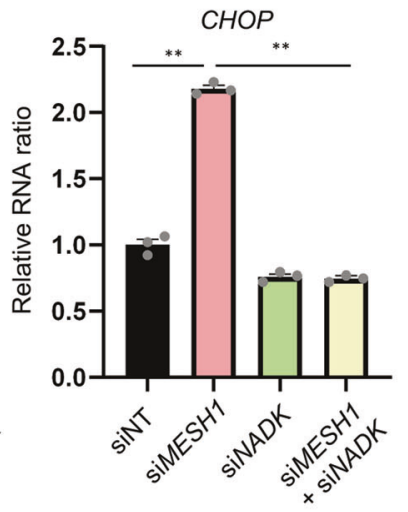

D

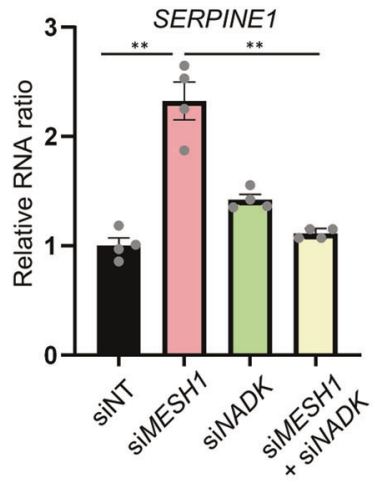

H

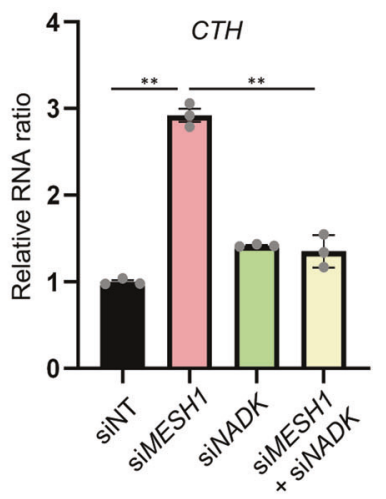

$\mathbf{K}$

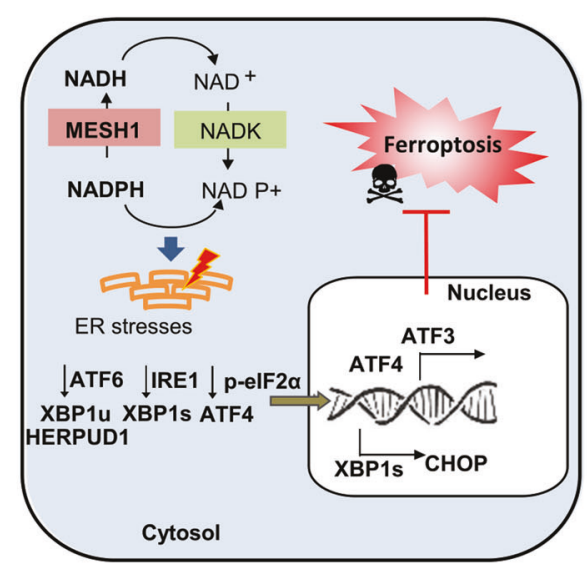

Fig. 5 The NADPH phosphatase activity of MESH1 regulates a subset of genes. A, B RCC4 cells transfected with non-targeting (NT) siRNA, MESH1-targeting siRNA, NADK-targeting siRNA, or both MESH1- and NADK-targeting siRNA were validated by its MESH1 (A) and NADK levels (B). C, D NADPH phosphatase activity of MESH1 regulates the mRNA level of VEGFA (C), and SERPINE1 (D). E-H the PERK pathway activated by MESH1 knockdown was shown to be mitigated by co-depletion of NADK as determined by WB of the elF2 $\alpha$ phosphorylation (E), ATF4 protein expression (E), and the mRNA levels of ATF3 (F), CHOP (G), CTH (H). I, J ATF6 target gene, total XBP1 (XBP1u) (I) and IRE1 target gene, spliced XBP1(XBP1s) (J) shown to be activated by MESH1 knockdown were also mitigated by concurrent knockdown of MESH1 and NADK. K Schematic illustration ISR triggered by MESH1 knockdown in preventing ferroptosis. Statistical analysis: ANOVA with Tukey HSD post hoc test, ${ }^{*} P<0.05$, **P $<0.01$.

results suggest that NADPH levels regulated by MESH1 may trigger ER stress and ISR as another mechanism to determine ferroptosis (Fig. 5K).

\section{DISCUSSION}

Here, we have shown that the MESH1 knockdown leads to a reproducible activation of the different branches of ER stresses, including PERK, IRE1, and ATF6. Therefore, MESH1 removal triggers ER stress and represents a form of mammalian stress response pathway.
Given the ability of MESH1 to dephosphorylate NADPH, the MESH1 knockdown has been shown to increase NADPH and protect ferroptosis [18]. Here, we found that NADK knockdown also abolishes the different features of the ER stress activation of $M E S H 1$ knockdown, suggesting a critical role of NADPH accumulation. Our results are consistent with several studies drawing the connections between NADPH and ISR or ER stress. For example, Hexose-6-phosphate dehydrogenase (H6PD) is an ER enzyme that generates NADPH and activates different arms of UPR, including ATF4, XBPs, and CHOP induction [33]. Another recent study also showed that elevated 
NADPH from the TCA cycles triggers the UPR in cancer cells as a metabolic sensing mechanism in ER [34]. Therefore, the accumulation of the NADPH upon MESH1 knockdown may also contribute to the ISR in the cancer cells. Such finding is also supported by an independent study of RSH in Caenorhabditis elegans [35]. However, the detailed mechanisms by which MESH1 removal and NADPH accumulation lead to ISR remains to be investigated in the future.

It is important to note that the MESH1 knockdown triggers a transcriptional program highly reminiscent of the transcriptional response of bacterial stringent response [1] and to Mesh1 removal in Drosophila [16]. Although MESH1 knockdown leads to activation of the ATF4 and ISR, these changes only contribute to $30 \%$ of the transcriptional response. Therefore, a significant portion of the transcriptional response to $\mathrm{MESH} 1$ knockdown was independent of ATF4. In addition to elF2a-ATF4, MESH1 depletion also activates the IRE1 and ATF6, indicating that MESH1 knockdown and NADPH accumulation activates all three branches of the ER stress response. Furthermore, the GO and GSEA analysis of the transcriptome responses to MESH1 knockdown showed the repression of the DNA proliferation, cell cycles, and mitotic pathways with significant similarities with the transcriptome response to the removal of Drosophila Mesh1 [16] and bacterial stringent response. Therefore, MESH1 removal may trigger a form of mammalian stringent-like stress response pathway beyond ER stress. An important distinction between the bacterial stringent response and the mammalian counterpart is that bacterial ppGpp is a signaling molecule whose level is dramatically induced during stress and binds to the RNA polymerase to mediate the transcriptome, whereas NADPH serves as an essential agent to mediate the lipid biosynthesis and regeneration of the GSH to overcome oxidative stresses. Together with previous studies $[16,18]$, these results suggest significant evolutional conservation of phenotypic and transcriptional response to the MESH1 removal across kingdoms.

MESH1 knockdown has been shown to confer resistance to ferroptosis induced by oxidative stresses [18]. The activation of ER stress has been shown to confer stress survival to various metabolic stresses $[15,20]$. For example, ATF4 upregulation confers the survival of amino acid deprivation [14], combined hypoxia and lactic acidosis [10, 13], and various tumor therapeutics [36, 37]. Therefore, we expect that MESH1 knockdown may enable stress survival under stress conditions beyond ferroptosis. While the role of MESH1 beyond ferroptosis remains to be established, its functional relevance and connection with other well-established stress responses will be actively investigated in the future.

\section{METHODS \\ Gene-silencing using RNAi}

RCC4 cells or 786-O cells were plated in a 6-well plate with $10^{5}$ cells per well. After $18 \mathrm{~h}$ of incubation, the cells were transfected with 40 pmol of siRNA and $3 \mu \mathrm{L}$ of Lipofectamine RNAiMAX for $72 \mathrm{~h}$. The efficacies of this siRNA were then validated by rt-qPCR or Western blots. Non-targeting siRNA (siNT) (Qiagen, AllStars Negative Control siRNA, SI03650318). Other siRNA includes siMESH1\#1 (target sequence GGGAAUCACUGACAUUGUG, D-031786-01, Dharmacon), siMESH1\#2 (target sequence CTGAAGGTCTCCTGCTAACTA, SI04167002, Qiagen), siATF4 (target sequence GAUCAUUCCUUUAGUUUAG, CAUGAUCCCUCAGUGCAUA, GUUUAGAGCUGGGCAGUGA, CUAGGUACCGCC AGAAGAA, M-005125-02, Dharmacon), siNADK (target sequence UGAAUGAGG UGGUGAUUGA, CGCCAGCGAUGAAAGCUUU, GAAGACGGCGUGCACAAU, CCA AUCAGAUAGACUUCAU, M-006318-01, Dharmacon). If not specified, siMESH1 indicated SIMESH1\#1.

\section{Cell culture and reagents}

The RCC4 cell line was a gift from Denise Chan (University of California, San Francisco, San Francisco, CA) and was authenticated by DDC (DNA Diagnostics Center) Medical using the short tandem repeat method and tested to be mycoplasma-free in November 2015. 786-O cells were purchased from the Cell Culture Facility at Duke University (Durham, NC, USA). 786-O cell line has been authenticated by STR DNA profiling and tested to be mycoplasma-free by Duke Cell Culture Facility. All cells were cultured in DMEM with $4.5 \mathrm{~g} / \mathrm{L}$ glucose and $4 \mathrm{mM}$ Glutamine (11995-DMEM, ThermoFisher Scientific) and 10\% heat-inactivated fetal bovine serum (Hyclone \# $\mathrm{SH} 30070.03 \mathrm{HI}$ ) in a humidified incubator, at $37^{\circ} \mathrm{C}$ with $5 \% \mathrm{CO}_{2}$. Tunicamycin (T7765) and brefeldin $A(B 7651)$ were purchased from Sigma. Erastin was synthesized at Small Molecule Synthesis Facility at Duke University.

\section{Western blots}

Western blotting was performed as previously described [38]. After $72 \mathrm{~h}$ of siRNA transfection, RCC4 cells were washed once with ice-cold PBS, then resuspended in RIPA buffer with protease and phosphatase inhibitors. The samples were then lysed by a constant vortex for $30 \mathrm{~min}$ at $4{ }^{\circ} \mathrm{C}$, then spun down at $13,000 \mathrm{rpm}$ for $10 \mathrm{~min}$ at $4{ }^{\circ} \mathrm{C}$. The protein concentration of the supernatant was determined by the Pierce BCA protein assay kit (\#23225, ThermoFisher). Around $40 \mu \mathrm{g}$ of the protein samples were loaded on $10 \%$ sodium dodecyl sulfate-polyacrylamide gel electrophoresis gels, transferred to PDVF membrane, blocked with $5 \%$ non-fat milk in TBST. The PVDF membranes were then incubated with primary antibodies overnight at $4{ }^{\circ} \mathrm{C}$. Primary antibodies: MESH1 (1:1000, HPA040895, Sigma); p-elF2a (1:1000, \#9721, Cell signaling); ATF4 (1:500,60035-1, Proteintech); a-tubulin (1:1000, sc32293, Santa Cruz); $\beta$-tubulin (1:1000, \#2128, Cell Signaling).

\section{Quantitative real-time PCR}

Total RNA from the samples was extracted using the RNeasy mini kit (Qiagen, 74104) following the manufacturer's instructions. Total RNA $(1.5 \mu \mathrm{g})$ was reverse transcribed to CDNA by random hexamers and SuperScript IV (Invitrogen). Power SYBRGreen Mix (ThermoFisher Scientific, 4367659) and StepOnePlus Real-time PCR system (Applied Biosystems) were then used to quantify relative RNA ratio. Samples were biologically triplicated for mean \pm SEM. The primer sequences for RT-qPCR are listed in Supplementary Table 3.

\section{Transcriptome analysis}

RNA quality was assessed using an Agilent BioAnalyzer (Agilent). cDNA library was prepared by Illumina TruSeq Stranded mRNA LT Sample Prep Kit - Set A (lllumina, RS-122-2101) according to the manufacturer's instructions. The library was pooled and sequenced using Illumina HiSeq 4000 with single-end $50 \mathrm{bp}$ read length at The Sequencing and Genomic Technologies Shared Resource of Duke Cancer Institute. The differential analysis was performed using DESeq2 [39]. For cDNA microarray, cDNA was amplified with Ambion MessageAmp Premier RNA Amplification kit (ThermoFisher Scientific, AM1792). The gene expression signatures were interrogated with Affymetrix U133A gene chips and normalized by the RMA (Robust Multi-Array) algorithm. CDNA synthesis and microarray interrogation were performed by the Duke Microarray Core.

\section{Cell viability and cytotoxicity assays}

Cell viability and cytotoxicity assays were performed as previously described [40, 41]. Cell viability of RCC4 cells was determined by CellTiter-Glo luminescent cell viability assay (Promega) following the manufacturer's protocol. After $48 \mathrm{~h}$ of siRNA transfection, RCC4 cells were treated with various doses of erastin. After another $20 \mathrm{~h}$ of incubation, CellTiter-Glo substrate $(15 \mu \mathrm{l})$ was added to the 96 -well plate with $100 \mu \mathrm{l}$ media for $10 \mathrm{~min}$ of continuous shaking. The cell viability was then determined by signal intensity using a chemiluminescence plate reader. Cytotoxicity assay was measured by the rupture of the cell membrane using CellTox Green cytotoxicity assay (Promega) by following the manufacturer's protocol. The fluorescent dye of the CellTox Green assay was added to the media (1:1000). After $30 \mathrm{~min}$ of incubation. The cytotoxicity of RCC4 cells as determined by membrane rupture was imaged by fluorescent microscopy and quantified by a fluorescence plate reader.

\section{Statistical analysis}

The number of biological replicates was presented by individual data points in each bar graph. $p$-values were determined by ANOVA with Tukey HSD post-hoc test in Graphpad. Error bars represent SEM, and significance between samples is denoted as ${ }^{*} p<0.05 ;{ }^{* *} p<0.01$.

\section{Data deposition}

The RNA-Seq and microarray data have been deposited into NCBI GEO with accession numbers: GSE114282 (RNAseq) and GSE114128 (cDNA microarray for siMESH1 and siATF4). 


\section{REFERENCES}

1. Potrykus K, Cashel M. (p)ppGpp: still magical? Annu Rev Microbiol. 2008;62:35-51.

2. Dalebroux ZD, Swanson MS. ppGpp: magic beyond RNA polymerase. Nat Rev Microbiol. 2012;10:203-12.

3. Diez S, Ryu J, Caban K, Gonzalez RL, Dworkin J. The alarmones (p)ppGpp directly regulate translation initiation during entry into quiescence. Proc Natl Acad. Sci USA 2020;117:15565-72.

4. Pollard JW, Lam T, Stanners CP. Mammalian cells do not have a stringent response. J. Cell Physiol. 1980;105:313-25.

5. Chi JT, Wang Z, Nuyten DS, Rodriguez EH, Schaner ME, Salim A, et al. Gene expression programs in response to hypoxia: cell type specificity and prognostic significance in human cancers. PLoS Med. 2006;3:e47.

6. Majmundar AJ, Wong WJ, Simon MC. Hypoxia-inducible factors and the response to hypoxic stress. Mol Cell. 2010;40:294-309.

7. Keenan MM, Liu B, Tang X, Wu J, Cyr D, Stevens RD, et al. ACLY and ACC1 regulate hypoxia-induced apoptosis by modulating ETV4 via alpha-ketoglutarate. PLoS Genet. 2015;11:e1005599.

8. Chen JL, Lucas JE, Schroeder T, Mori S, Wu J, Nevins J, et al. The genomic analysis of lactic acidosis and acidosis response in human cancers.PLoS Genet. 2008;4:e1000293.

9. Chen JLY, Merl D, Peterson CW, Wu J, Liu PY, Yin H, et al. Lactic acidosis triggers starvation response with paradoxical induction of TXNIP through MondoA. PLOS Genet. 2010;6:e1001093. https://doi.org/10.1371/journal.pgen.1001093.

10. Tang $X$, Lucas JE, Chen JL, LaMonte G, Wu J, Wang MC et al. Functional interaction between responses to lactic acidosis and hypoxia regulates genomic transcriptional outputs. Cancer Res. 2012;72:491-502.

11. Lamonte G, Tang X, Chen JL, Wu J, Ding CK, Keenan MM, et al. Acidosis induces reprogramming of cellular metabolism to mitigate oxidative stress. Cancer Metab. 2013;1:23.

12. Hardie DG, Ross FA, Hawley SA. AMPK: a nutrient and energy sensor that maintains energy homeostasis. Nat. Rev. Mol. Cell Biol. 2012;13:251-62.

13. Lucas JE, Kung HN, Chi JT. Latent factor analysis to discover pathway-associated putative segmental aneuploidies in human cancers. PLoS Comput Biol. 2010;6: e1000920.

14. Tang X, Keenan MM, Wu J, Lin CA, Dubois L, Thompson JW, et al. Comprehensive profiling of amino acid response uncovers unique methionine-deprived response dependent on intact creatine biosynthesis. PLoS Genet. 2015;11:e1005158.

15. Pakos-Zebrucka K, Koryga I, Mnich K, Ljujic M, Samali A, Gorman AM. The integrated stress response. EMBO Rep. 2016;17:1374-95.

16. Sun D, Lee G, Lee JH, Kim HY, Rhee HW, Park SY, et al. A metazoan ortholog of SpoT hydrolyzes ppGpp and functions in starvation responses. Nat. Struct. Mol. Biol. 2010;17:1188-94.

17. Ding C-KC, Rose J, Wu J, Sun T, Chen K-Y, Chen P-H, et al. Mammalian stringentlike response mediated by the cytosolic NADPH phosphatase MESH1. Preprint at bioRxiv https://doi.org/10.1101/325266 (2018).

18. Ding C-KC, Rose J, Sun T, Wu J, Chen P-H, Lin C-C, et al. MESH1 is a cytosolic NADPH phosphatase that regulates ferroptosis. Nat Metab. 2020;2:270-7.

19. Lin CC, Chi JT. Ferroptosis of epithelial ovarian cancer: genetic determinants and therapeutic potential. Oncotarget 2020;11:3562-70.

20. Chen PH, Wu J, Xu Y, Ding CC, Mestre AA, Lin CC, et al. Zinc transporter ZIP7 is a novel determinant of ferroptosis. Cell Death Dis. 2021;12:198.

21. Ferullo DJ, Lovett ST. The stringent response and cell cycle arrest in Escherichia coli. PLoS Genet. 2008;4:e1000300.

22. Durfee T, Hansen AM, Zhi H, Blattner FR, Jin DJ. Transcription profiling of the stringent response in Escherichia coli. J Bacteriol. 2008;190:1084-96.

23. Nazir A, Harinarayanan R. (p)ppGpp and the bacterial cell cycle. J Biosci. 2016;41:277-82.

24. Vattem KM, Wek RC. Reinitiation involving upstream ORFs regulates mRNA translation in mammalian cells. Proc Natl Acad Sci USA. 2004;101:11269-74.

25. Kober L, Zehe C, Bode J. Development of a novel ER stress based selection system for the isolation of highly productive clones. Biotechnol Bioeng. 2012;109:2599-611.

26. Wyszynski FJ, Lee SS, Yabe T, Wang H, Gomez-Escribano JP, Bibb MJ, et al. Biosynthesis of the tunicamycin antibiotics proceeds via unique exo-glycal intermediates. Na. Chem. 2012;4:539-46.

27. Lee YS, Kalimuthu K, Seok Park Y, Makala H, Watkins SC, Choudry MHA. et al. Ferroptotic agent-induced endoplasmic reticulum stress response plays a pivotal role in the autophagic process outcome. J Cell Physiol. 2020;235:6767-78. https:// doi.org/10.1002/jcp.29571.

28. Chen $Y$, Mi Y, Zhang X, Ma Q, Song Y, Zhang L, et al. Dihydroartemisinin-induced unfolded protein response feedback attenuates ferroptosis via PERK/ATF4/HSPA5 pathway in glioma cells. J Exp Clin Cancer Res. 2019;38:402.

29. Zhu S, Zhang Q, Sun X, Zeh HJ 3rd, Lotze MT, Kang R, et al. HSPA5 regulates ferroptotic cell death in cancer cells. Cancer Res. 2017;77:2064-77.
30. Helms JB, Rothman JE. Inhibition by brefeldin A of a Golgi membrane enzyme that catalyses exchange of guanine nucleotide bound to ARF. Nature 1992;360:352-4.

31. Gerdes SY, Scholle MD, D'Souza M, Bernal A, Baev MV, Farrell M, et al. From genetic footprinting to antimicrobial drug targets: examples in cofactor biosynthetic pathways. J Bacteriol. 2002;184:4555-72.

32. Kriel A, Bittner AN, Kim SH, Liu K, Tehranchi AK, Zou WY, et al. Direct regulation of GTP homeostasis by (p)ppGpp: a critical component of viability and stress resistance. Mol Cell. 2012;48:231-41.

33. Tsachaki M, Mladenovic N, Stambergova H, Birk J, Odermatt A. Hexose-6phosphate dehydrogenase controls cancer cell proliferation and migration through pleiotropic effects on the unfolded-protein response, calcium homeostasis, and redox balance. FASEB J. 2018;32:2690-705.

34. Gansemer ER, McCommis KS, Martino M, King-McAlpin AQ, Potthoff MJ, Finck BN, et al. NADPH and glutathione redox link TCA cycle activity to endoplasmic reticulum homeostasis. iScience. 2020;23:101116.

35. Mak KH, Zhao Q, Hu P-W, Au-Yeung C-L, Yang J, Duraine L, et al. Lysosomal nucleotide metabolism regulates ER proteostasis through mTOR signaling. Preprint at bioRxiv https://doi.org/10.1101/2020.04.18.048561 (2020).

36. Shi Z, Yu X, Yuan M, Lv W, Feng T, Bai R, et al. Activation of the PERK-ATF4 pathway promotes chemo-resistance in colon cancer cells. Sci Rep. 2019;9:3210.

37. Madden $E$, Logue SE, Healy SJ, Manie S, Samali A. The role of the unfolded protein response in cancer progression: from oncogenesis to chemoresistance. Biol Cell. 2019;111:1-17.

38. Lin CC, Kitagawa M, Tang X, Hou MH, Wu J, Qu DC, et al. CoA synthase regulates mitotic fidelity via CBP-mediated acetylation. Nat Commun. 2018;9:1039.

39. Love MI, Huber W, Anders S. Moderated estimation of fold change and dispersion for RNA-seq data with DESeq2. Genome Biol. 2014;15:550.

40. Lin CC, Yang WH, Lin YT, Tang X, Chen PH, Ding CC, et al. DDR2 upregulation confers ferroptosis susceptibility of recurrent breast tumors through the Hippo pathway. Oncogene 2021;40:2018-34.

41. Lin CC, Mabe NW, Lin YT, Yang WH, Tang X, Hong L, et al. RIPK3 upregulation confers robust proliferation and collateral cystine-dependence on breast cancer recurrence. Cell Death Differ. 2020;27:2234-47.

\section{ACKNOWLEDGEMENTS}

We thank members of Chi lab for critical feedback and technical assistance.

\section{AUTHOR CONTRIBUTIONS}

$\mathrm{CCL}, \mathrm{CDK}$, TS, and JW performed the experiments. KYC performed the data analysis. $\mathrm{CCL}, \mathrm{CDK}, \mathrm{PZ}$, and JTC wrote the paper. JTC and PZ designed and conceptualized the study.

\section{FUNDING}

This work was supported by NIH (R01GM124062) and Duke Bridge Funding, DCI Pilot project (to JTC and PZ), and DOD (W81XWH-17-1-0143, W81XWH-15-1-0486, W81XWH-20-1-0907) and NIH (1R01NS111588) and Emerson Foundation to JTC.

\section{COMPETING INTERESTS}

The authors declare no competing interests.

\section{CONSENT FOR PUBLICATION}

All authors have provided their consent for publication.

\section{ADDITIONAL INFORMATION}

Supplementary information The online version contains supplementary material available at https://doi.org/10.1038/s41419-021-04018-7.

Correspondence and requests for materials should be addressed to J.-T.C.

Reprints and permission information is available at http://www.nature.com/ reprints

Publisher's note Springer Nature remains neutral with regard to jurisdictional claims in published maps and institutional affiliations. 
(c) (i) Open Access This article is licensed under a Creative Commons Attribution 4.0 International License, which permits use, sharing, adaptation, distribution and reproduction in any medium or format, as long as you give appropriate credit to the original author(s) and the source, provide a link to the Creative Commons license, and indicate if changes were made. The images or other third party material in this article are included in the article's Creative Commons license, unless indicated otherwise in a credit line to the material. If material is not included in the article's Creative Commons license and your intended use is not permitted by statutory regulation or exceeds the permitted use, you will need to obtain permission directly from the copyright holder. To view a copy of this license, visit http://creativecommons. org/licenses/by/4.0/.

(c) The Author(s) 2021 